\title{
The Effects of Cross-Hemispheric Dorsolateral Prefrontal Cortex Transcranial Direct Current Stimulation (tDCS) on Task Switching
}

\author{
Jorge Leite ${ }^{\mathrm{a}, *}$, Sandra Carvalho ${ }^{\mathrm{a}}$, Felipe Fregni ${ }^{\mathrm{b}}$, Paulo S. Boggio ${ }^{\mathrm{c}}$, Óscar F. Gonçalves ${ }^{\mathrm{a}}$ \\ a Neuropsychophysiology Laboratory, CIPsi, School of Psychology (EPsi), University of Minho, Campus de Gualtar, $4710-057$ Braga, Portugal \\ ${ }^{\mathrm{b}}$ Neuromodulation Laboratory, Spaulding Rehabilitation Hospital, Harvard Medical School, Boston, MA, USA

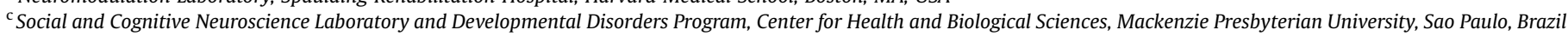

\section{A R T I C L E I N F O}

\section{Article history:}

Received 11 February 2012

Received in revised form

21 September 2012

Accepted 14 October 2012

Available online 9 November 2012

\section{Keywords:}

Task switching

PFC

Cross-hemispheric tDCS

\begin{abstract}
A B S T R A C T
Background: Task switching, defined as the ability to flexibly switch between tasks in the face of goal shifting, is a central mechanism in cognitive control. Task switching is thought to involve both prefrontal cortex (PFC) and parietal regions. Our previous work has shown that it is possible to modulate set shifting tasks using $1 \mathrm{~mA}$ tDCS on both the left dorsolateral prefrontal cortex and the left primary motor area. However, it remains unclear whether the effects of PFC tDCS on task switching are hemisphere-dependent. Objectives: We aimed to test the effects of three types of cross-hemispheric tDCS over the PFC (left anode-right cathode [LA-RC], left cathode-right anode [LC-RA] and sham stimulation) on participants' performance (reaction time) and accuracy (correct responses) in two task-switching paradigms (i.e., letter/digit naming and vowel-consonant/parity tasks).

Methods: Sixteen participants received cross-hemispheric tDCS over the PFC in two task-switching paradigms.

Results: The results show that cross-hemispheric tDCS over the PFC modulates task-switching ability in both paradigms. Our results were task and hemisphere-specific, such that in the letter/digit naming task, LA-RC tDCS increased switching performance, whereas LC-RA tDCS improved accuracy. On the other hand, in the vowel-consonant/parity task, LA-RC improved accuracy, and decreased switching performance.

Conclusions: Our findings confirm the notion that involvement of the PFC on task switching depends critically on laterality, implying the existence of different roles for the left hemisphere and the right hemisphere in task switching.
\end{abstract}

(c) 2013 Elsevier Inc. All rights reserved.

\section{Introduction}

The ability to flexibly switch between tasks when changes in the goal state occur [1] is considered to be a central mechanism in cognitive control and behavior flexibility. Task switching (see [2] for review) has been found to be dependent on a broader frontoparietal brain network [3] with higher parietal activation, particularly in situations with high shift costs [4].

Several neuroimaging studies have also associated prefrontal cortex (PFC) regions with task switching, particularly in situations

Author's notes: Special authorship status-Jorge Leite and Sandra Carvalho share co-first authorship.

This work was supported by the Portuguese Foundation for Science and Technology with individual grants (SFRH/BD/41484/2007 and SFRH/BD/64355/2009).

Conflict of interest: The authors do not have any conflicts of interest, financial or otherwise, to disclose.

* Corresponding author. Tel.: +351 253604220, fax: +351 253604224

E-mail address: jorgel@psi.uminho.pt (J. Leite). that require some type of rule shifting (see [5] for a detailed explanation). Frontal regions are thought to be involved in conditions requiring the inhibition of a previously acquired prepotent response (i.e., top-down conflict reduction), with the parietal cortex playing a role in the reconfiguration of the stimulus-response (S-R) mappings [6]. Patients with a lesioned PFC frequently show impairments in task-switching ability [7].

Taken together, experimental and clinical evidence has shown that the endogenous preparation and the exogenous executive adjustments required in task switching depend on both PFC and parietal regions [8]. Despite this evidence, little is known about the differential contributions of each hemisphere, particularly in the PFC.

Our previous work [9] has shown that it is possible to modulate set shifting tasks using "offline" (i.e., the stimulation occurs immediately before the task performance) $1 \mathrm{~mA}$ transcranial direct current stimulation (tDCS) on both the dorsolateral prefrontal cortex (DLPFC) and the primary motor area (M1). This study only tested unilateral PFC (left hemisphere) tDCS. However, data on 
functional asymmetry between the right and left PFC (e.g., working memory rehearsal/maintenance) [10] supports the need to test the effects of cross-hemispheric PFC tDCS.

The interaction between the homotopic PFC regions may be of particular interest because the left and the right PFC seem to have distinct strategies for information processing. Whereas the left hemisphere (LH) seems to act as an interpreter in deciding ambiguous conditions (e.g., [11]), the right hemisphere (RH) can accommodate more ambiguous situations [12]. In fact, studies with patient populations showed that patients with RH lesions are more prone to errors when the information is indeterminate and insufficient, whereas patients with LH lesions perform worse in situations in which the information is complete [13]. Also studies with working memory (WM) suggest the involvement of both PFC hemispheres: anodal tDCS over the left dorsolateral PFC is able to increase WM performance [14], but neuroimaging studies suggest also the involvement of the $\mathrm{RH}$ when there are increases in the WM load demand [15].

Cross-hemispheric tDCS allows modulation of the PFC activity, in such manner that the anode increases the activity in the underlying region [16], while cathodal tDCS over the homotopic contralateral region decreases the activity [17].

The present study uses two visual cue switch tasks, with similar S-R mappings, but with different levels of cognitive demand. The first task is a letter/digit naming task, with fixed, clear and defined visual stimulus and motor response (S-R) mappings. The second task, the vowel-consonant/parity task, relies on the same S-R mappings of the previous one, but demands a secondary task (i.e., a judgment of the visual target as vowel-consonant or as odd-even) prior to response selection.

Therefore, the objective of this study is to test the effects of three types of cross-hemispheric tDCS over the PFC (i.e., left anode-right cathode [LA-RC], left cathode-right anode [LC-RA] and sham) on participants' performance (i.e., reaction time) and accuracy (i.e., correct responses) in two task-switching paradigms (i.e., letter/digit naming and vowel-consonant/parity tasks). In summary, the present study aims to modulate the activity of both right and left PFC, simultaneously and in opposite directions, assessing the effects in two task-switching behavioral tasks.

\section{Methods}

\section{Participants}

Sixteen college student volunteers (age: $24 \pm 7.702,13$ females) participated in the study. All participants were right-handed and healthy, with normal or corrected-to-normal visual acuity and without present or past history of neurological or psychiatric disorders. Participants were excluded if any medication or psychotropic drugs had been used during the 4 weeks prior to the study. Participants were advised to avoid alcohol, cigarettes and caffeinated drinks on the day of the experiment, and none reported fatigue due to insufficient sleep.

All participants gave their written informed consent prior to their inclusion in the study. The study was approved by the local ethics committee and was in accordance with the Declaration of Helsinki.

\section{Procedure}

\section{Experimental design}

Each session started with $3 \mathrm{~min}$ of tDCS applied to each participant prior to execution of the control tasks [18], an interval that has been reported to be the minimum amount of time required to produce tDCS after-effects [19]. Then, each experimental session started with a simple reaction time task followed by a choice reaction time for both the left and right index fingers. There was an interval of $1 \mathrm{~min}$ between the simple and choice reaction time tasks. The letter/digit naming and the vowel-consonant/parity tasks were then carried out, with a 2 min interval between them. The order of the experimental tasks was fully randomized and counterbalanced across participants and sessions.

At the end of each session, participants responded to a brief questionnaire checking if the participants felt the tDCS, existence of mood changes or report of any adverse effect. In the last session, participants were also asked if they were aware of the identical key bindings across tasks.

Letter/digit naming task. This task consisted of a pair of targets (i.e., a letter and a digit) that always appeared in the center of the screen with an approximately $0.6^{\circ}$ visual angle. Surrounding those targets, a colored circle (green or red) with an approximately $3^{\circ}$ visual angle was presented. This color served as a visual cue to the participant, signaling the response that he was required to perform: if the color was green, the participant was required to respond to the letter, whereas if it was red, a response to the digit was required.

Each session was preceded by a training phase consisting of 32 trials in which the participant was required to perform with $100 \%$ accuracy before proceeding to the task. In the training phase, the participants were instructed that if the cue was green and the letter presented on screen was an A, they should press the " $Z$ " key with the middle finger of the left hand. If the letter presented was an $\mathrm{H}$, they should press the " $X$ " key with the index finger of the left hand. If the cue was red, participants were required to respond to the digit. If the number was 4 or 9 , participants should use the index finger or the middle finger of the right hand on the " $\mathrm{N}$ " or " $\mathrm{M}$ " key, respectively. Each trial started with a fixation point in the center of the screen that lasted for $500 \mathrm{~ms}$; immediately after, the pair of targets appeared on screen and remained for $2500 \mathrm{~ms}$. If no response was detected, or in the event of an incorrect response, a screen that lasted for 1000 ms appeared with the word "Incorrect" with a hint for the correct response.

After training, participants performed the experimental task consisting of 128 trials of paired targets (32 trials for each letter/ digit-A, H, 4 or 9). Each trial started with a fixator at the center of the screen that lasted for 500 ms. Then the first pair of targets (consisting always of a letter and a digit) (TP1) would appear, remaining on screen up until a key press or until the $2500 \mathrm{~ms}$ time limit; immediately after, the second pair of targets (TP2) appeared on screen with the same time limit (as depicted in Fig. 1). After either a key press or the time limit, the trial ended, and the fixator for the new trial appeared on screen. This task had a maximum duration of $13 \mathrm{~min}$ (if no key presses were detected).

Vowel-consonant/parity task. This task was identical to the digit naming task except that the response required a judgment. In the green cue condition, participants were requested to respond to the letter, by pressing the " $Z$ " key with the left middle finger for vowels and the " $X$ " key with the left index finger for consonants. In the red cue condition, participants were required to respond to the number by pressing the " $\mathrm{N}$ " or "M" key with the right index or middle fingers if the number was even or odd, respectively. An additional $20 \%$ of trials consisted of pairs of novel targets (e.g., 5 and E), which were analyzed separately as they did not have an equivalent trial in the letter/digit naming task. They were inserted to mask the fact that both tasks were identical in terms of response selection; therefore, these pairs of novel targets were inserted to mask the fact that the main tasks could be solved by using the same strategy (i.e., associating the letter or the digit to the same key binding). This task had a maximum duration of $15 \mathrm{~min}$. 
Letter/Digit Naming Task

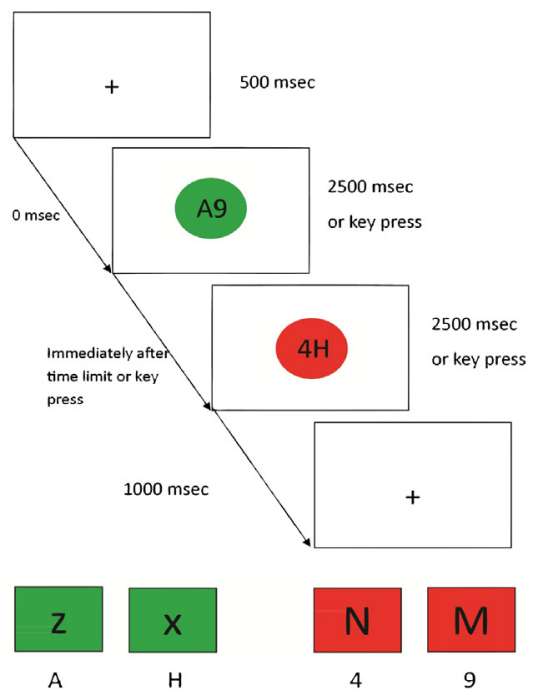

Vowel-Consonant/Parity Task

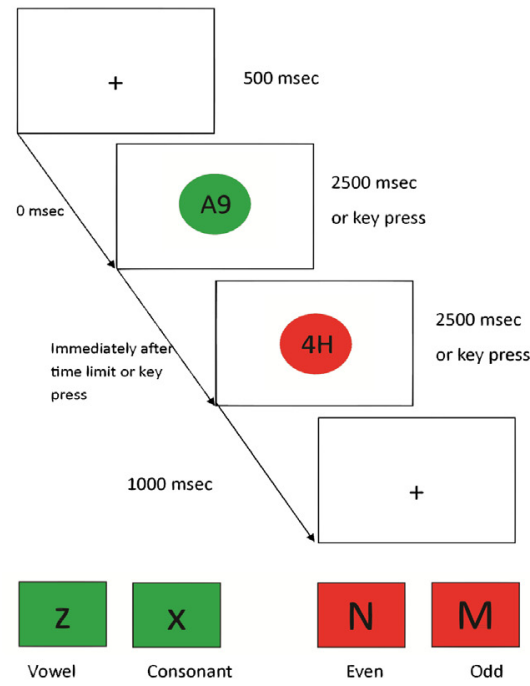

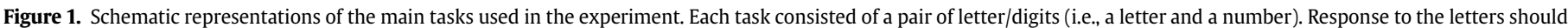

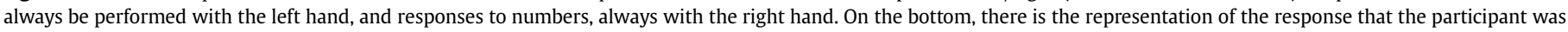
required to perform, with the associated key press.

For both tasks, in each trial that consisted of two consecutive paired targets, every time, the cue color remained the same it was considered a repeat trial; if not, it was a switch trial. There was a $50 \%$ probability of a switch or repeat trial. Letter/digit location was also randomized and letters and numbers had a 50\% probability of appearing on the left side of the pair (e.g., H9 and 9H).

For both tasks, only correct responses were submitted for further analysis. The remaining responses were considered errors and their RTs were not included in the estimates.

Control tasks. In order to control for possible motor or response speed changes due to tDCS, simple and choice reaction time tasks were performed.

Simple reaction time. In these tasks, participants were requested to respond as quickly as possible to a target appearing in the center of the screen. There were a total of 32 trials for each task. For each trial, a fixator appeared on the screen for a period of 200, 400, 600 or $800 \mathrm{~ms}$ (jittered) being then replaced by the target. Immediately after the response, a blank screen appeared for $500 \mathrm{~ms}$. Participants performed two different tasks, each one requiring only one target. If the target was “*”, the participant was required to press the " $\mathrm{X}$ " key with the index finger of the left hand. In the other task, "\#" served as a target, and the participant was required to press the " $\mathrm{N}$ " key with the index finger of the right hand. The order of these tasks (i.e., left and right) was fully randomized and counterbalanced between participants and sessions. These tasks had an approximate duration of 2 min.

Choice reaction time. In the choice reaction time, participants were requested to respond as quickly as possible to the target as soon as it appeared in the center of the screen. There were a total of 64 trials. For every trial, a fixator appeared at the center of the screen, and remained there for $200,400,600$ or $800 \mathrm{~ms}$ (jittered) being then replaced by the target. Immediately after the participant's response, a blank screen appeared for $500 \mathrm{~ms}$ followed by one of two possible targets. If "*” appeared on screen, the participant was required to press the " $X$ " key with the index finger of the left hand. If "\#" appeared, the participant had to press the "N" key with the right hand. There was a $50 \%$ probability for each target. This task had an approximate duration of $2 \mathrm{~min}$.
tDCS parameters. Two homotopic regions were selected as cortical targets: left PFC (F3) and right PFC (F4), and the tDCS was delivered by a battery-driven Eldith Stimulator DC+ (Neuroconn, Germany) using $2 \mathrm{~mA}$ (15 s ramp up and then $15 \mathrm{~s}$ down) with $35 \mathrm{~cm}^{2}$ salinesoaked sponge electrodes (current density of $0.057 \mathrm{~mA} / \mathrm{cm}^{2}$ ). tDCS was delivered for the entire duration of the experiment (maximum duration of $30 \mathrm{~min}$, as none of the participants reached the maximum duration of each task) and started 3 min before the beginning of the control tasks. The active electrodes were placed over F3 and F4 according to the 10-20 electrode system [20].

Left anode-right cathode (LA-RC) consisted of placing the anode over the left PFC and the cathode over the right PFC. Conversely, left cathode-right anode (LC-RA) consisted of placing the cathode over the left PFC and the anode over the right PFC. The sham condition was performed with an LA-RC electrodes configuration and consisted of a $15 \mathrm{~s}$ ramp up, $15 \mathrm{~s}$ of plateau and then $15 \mathrm{~s}$ down with the electrodes remaining on the head for the entire duration of the task.

All participants were subjected to the three tDCS conditions. Therefore, the polarity of tDCS was randomized and counterbalanced across participants, and the washout period was $24 \mathrm{~h}$ between the first and the second sessions and $48 \mathrm{~h}$ between the second and third sessions. This was performed to allow participants to integrate the experiment, as a part of their course.

\section{Data analysis}

To test the effects of tDCS in the simple reaction time task, repeated measures ANOVAs were performed with two factors: tDCS (three levels: LA-RC, LC-RA and SHAM) and hand (two levels: left and right).

Repeated measures ANOVAs were also performed to test: a) the effects of tDCS in the choice reaction time task with tDCS as a factor; b) the effect of tDCS subsequent to switched versus repeated trials including the following factors: switched and repeated, task (letter/ digit naming and vowel-consonant/parity) and tDCS; c) the effects of tDCS in the difference in terms of reaction times (RTs) between switched and repeated trials (i.e., switch cost performance), including the following factors: tDCS and task; d) the effects of tDCS in the difference in terms of accuracy between switched and repeated trials (i.e., switch cost error), including the following 
factors: tDCS and task; e) the overall accuracy when performing the task independently of being a switch or a repeat trial with the factors tDCS and task. For all models, we also tested the respective interaction effects. An exploratory analysis for the novel targets was also performed, including the effects of tDCS in (switch/repeat and tDCS as factors), as well as three one way repeated measures ANOVAs, exploring the effects of tDCS in terms of switch costs, accuracy and switch cost errors, respectively.

When sphericity was not met, the Greenhouse-Geisser correction was applied to degrees of freedom in all cases with the corrected probabilities and partial eta-squared $\left(\eta_{\mathrm{p}}^{2}\right)$ statistic reported. Effect size $f(f)$ and observed power (pwr) (computed with an alpha of 0.05 ) are also reported. Post-hoc comparisons of the mean values were carried out by paired multiple comparisons (Fischer LSD) when ANOVA revealed significant effects. The criterion for statistical significance was established at $P<.05$. All statistical analyses were performed with SPSS for Windows (version 20.0.0, IBM, US).

Data are presented as mean $(M)$ and standard error (SE). To address possible outliers, we established a cutoff in which all scores over 2 standard deviations from the mean were removed (which represents less than $5 \%$ of the total number of scores).

\section{Results}

None of the participants in this study reported mood alterations due to stimulation or experienced any adverse effects. In the sham condition, participants reported a tingling sensation similar to the one reported in the active tDCS conditions. None of the participants reported awareness of the identical key bindings across tasks. The Results section will present (i) the data on the control tasks (RTs and accuracy); (ii) switch/repeat performance in terms of RTs; (iii) switch cost performance, measured by the switch costs in terms of RTs (i.e., time difference between a switch and a repeat trial), and switch cost errors (i.e., error percentage difference between the switch and the repeat trials) for both the letter/digit naming and the vowel-consonant/parity tasks; (iv) the overall accuracy for the letter/digit naming and vowel-consonant/parity tasks (i.e., the percentage of correct responses, independently of being a switch or a repeat trial, is used as an overall index of accuracy during task performance); and (v) exploratory analysis of the novel targets in the vowel-consonant/parity task. All of the results are presented in terms of Mean $(M)$ and Standard Error (SE).

\section{Control tasks}

Simple reaction time

There were no statistically significant differences in RTs due to the hand (left or right) $\left(F(1,15)=.379, P>.05, \eta_{\mathrm{p}}^{2}=.025, f=.160\right.$, pwr $=.110), \operatorname{tDCS}\left(F(2,30)=.406, P>.05, \eta_{\mathrm{p}}^{2}=.026, f=.163\right.$, pwr $=.089)$ or the interaction between tDCS and the hand $\left(F(2,30)=.746, P>.05, \eta_{\mathrm{p}}^{2}=.047, f=.222\right.$, pwr $\left.=.165\right)($ see Fig. 2).

\section{Choice reaction time: $R T$ and accuracy}

For the choice reactions, there was no statistically significant main effect of tDCS on RT $\left(F(2,30)=.274, P>.05, \eta_{\mathrm{p}}^{2}=.018, f=.135\right.$, $\mathrm{pwr}=.089)$ or accuracy $\left(F(2,30)=2.043, P>.05, \eta_{\mathrm{p}}^{2}=.120, f=.369\right.$, $\mathrm{pwr}=.387)$.

Interaction between tDCS and task: performance, switch error and accuracy

\section{Effects of tDCS in reaction times subsequent to switched and repeated trials}

There was a main effect of switch/repeat for both tasks $\left(F(1,15)=429.766, P<.001, \eta_{\mathrm{p}}^{2}=.966, f=5.330, \mathrm{pwr}=1.000\right)$, with the repeat trials significantly decreasing the RT $(M=680.40$, $\mathrm{SE}=33.052)$ when comparing to switch trials $(M=1039.032$, $\mathrm{SE}=43.737)(P<.001)$. Also, there was a main effect of task $\left(F(1,15)=25.491, P<.001, \eta_{\mathrm{p}}^{2}=.630, f=1.305, \mathrm{pwr}=.997\right)$, with shorter RT in the letter/digit naming task $(M=819.353$, $\mathrm{SE}=37.392)$ when comparing to the vowel-consonant/parity task $(M=900.139, \mathrm{SE}=39.819)(P<.001)$. No main effects of tDCS were found $\left(F(2,30)=.243, P>.05, \eta_{\mathrm{p}}^{2}=.016, f=.128, \mathrm{pwr}=.085\right)$. From all the possible interaction in the model, only the interaction between switch/repeat, task and tDCS was significant $\left(F(2,30)=10.548, P<.001, \eta_{\mathrm{p}}^{2}=.413, f=.839, \mathrm{pwr}=.981\right)$, but the post hoc testing revealed that the effects were explained by the main effect of switch/repeat and task (see Fig. 4).

\section{Effects of tDCS in switch cost performance: difference in RTs between switch and repeat trials}

There was a significant interaction between tDCS and the task $\left(F(2,30)=12.047, P<001, \eta_{\mathrm{p}}^{2}=.445, f=.895, \mathrm{pwr}=.991\right)$ on RT, without main effect of task $\left(F(1,15)=.202, P>.05, \eta_{\mathrm{p}}^{2}=.013\right.$, $f=.115$, pwr $=.071)$. The post-hoc pairwise comparison revealed that for the letter/digit naming task, LA-RC $(M=318.542$, $\mathrm{SE}=19.948)$ significantly decreased the RT compared to both LC-RA $(M=378.081, \mathrm{SE}=19.434)(P=.001)$ and sham $(M=373.307$, $\mathrm{SE}=24.507)(P=.033)$. No differences were found between LC-RA and sham $(P>.05)$. For the vowel-consonant/parity task, LA-RC $(M=399.358, \mathrm{SE}=23.233)$ significantly increased the RT compared to both LC-RA $(M=340.268, \mathrm{SE}=21.235)(P=.003)$ and sham $(M=347.658, \mathrm{SE}=23.041)(P=.044)$. No statistically significant differences were found between sham and LC-RA $(P>.05)$ (see Fig. 3).
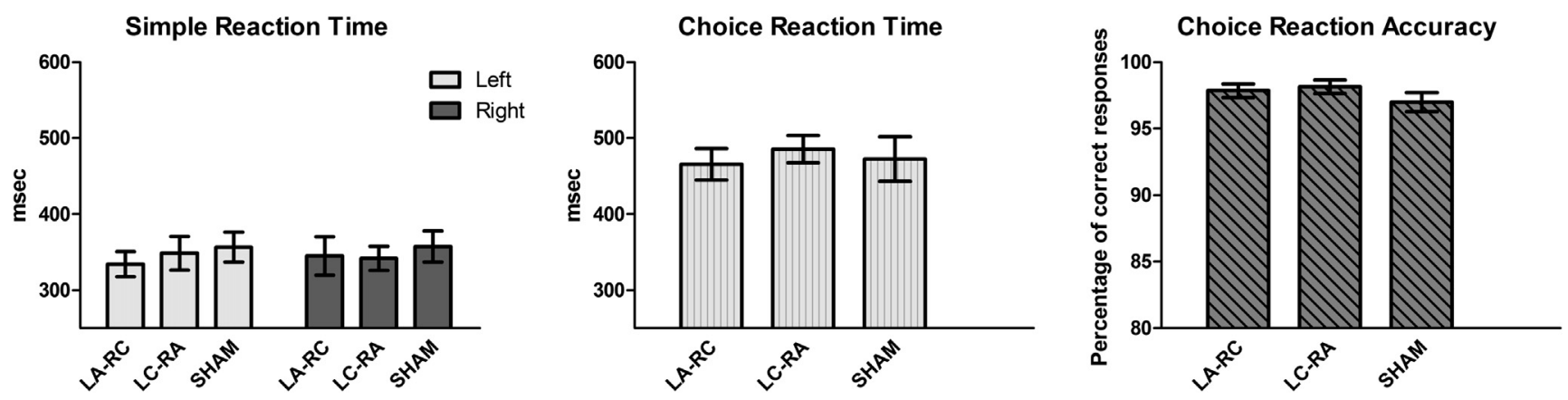

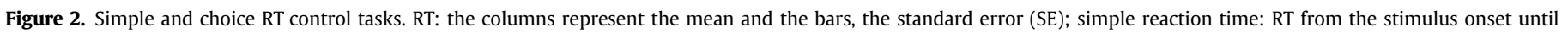

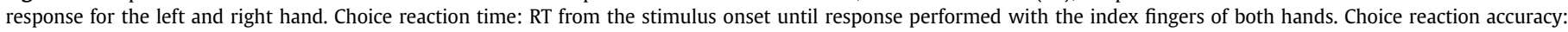
overall percentage of correct responses. 
Switch Cost Performance

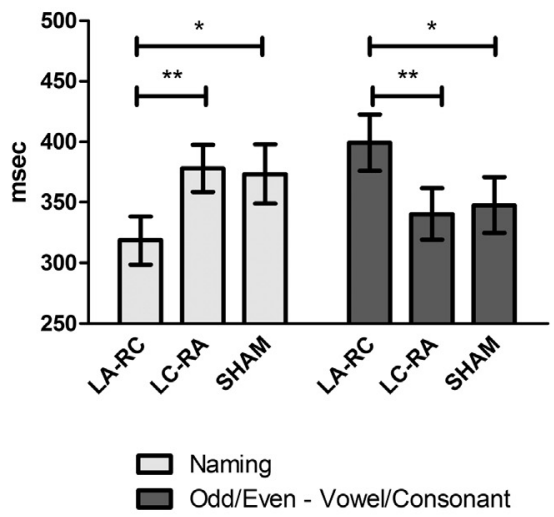

Switch Cost Errors

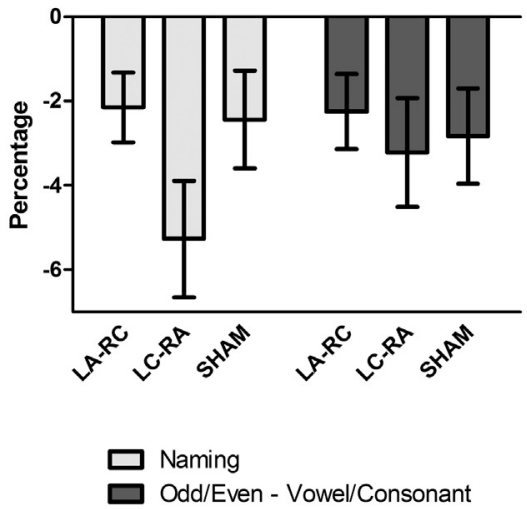

Overall accuracy

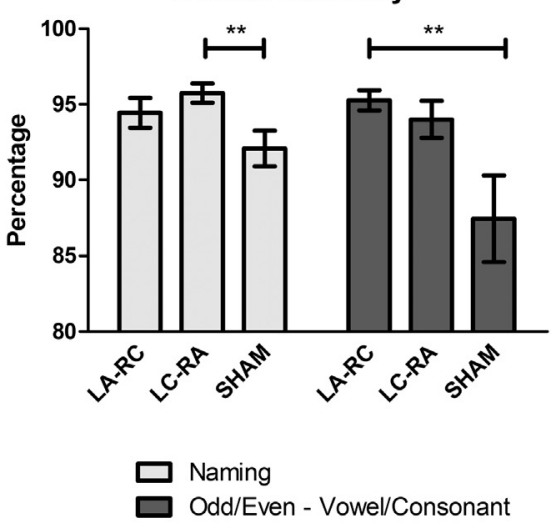

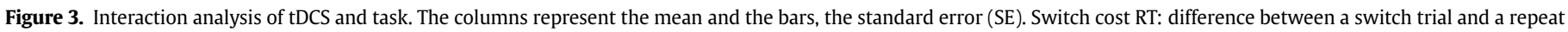

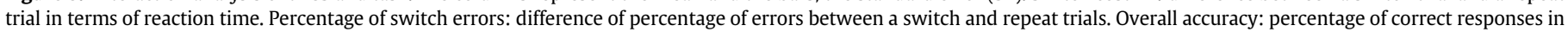
performing the task (including the switch and repeat trials). ${ }^{*} P<.05 ;{ }^{* *} P<.01$; ${ }^{* * *} P<.001$.

The tDCS effects across tasks show that both sham and LC-RA produce similar results in terms of switch cost RTs, independently of the task $(P>.05)$. On the contrary, LA-RC produces significant alterations in switch costs depending on the task $(P=.001)$.

Effects of tDCS in accuracy between switch and repeat trials: switch cost errors

There was no statistically significant interaction between tDCS and tasks in terms of switching cost errors $(F(2,30)=.657, P>.05$, $\eta_{\mathrm{p}}^{2}=.042, f=.209$, pwr $=.150$ ) (see Fig. 3 ) or across tasks $\left(F(1,15)=.408, P>.05, \eta_{\mathrm{p}}^{2}=.026, f=.163\right.$, pwr $\left.=.092\right)$.

Effect of tDCS in overall accuracy independently of being a switched or a repeated trial

There was a statistically significant interaction between tDCS and tasks $\left(F(2,30)=3.515, P=.043, \eta_{\mathrm{p}}^{2}=.190, f=.484\right.$, pwr $\left.=.611\right)$, without main effect of the task $\left(F(1,15)=2.938, P>.05, \eta_{\mathrm{p}}^{2}=.164\right.$, $f=.443$, pwr $=.361)$. The post-hoc pairwise comparison revealed that, for the letter/digit naming task, $\mathrm{LC}-\mathrm{RA}(M=95.752, \mathrm{SE}=.634)$ significantly increased accuracy compared to sham $(M=92.091$, $\mathrm{SE}=1.191)(P=.007)$. No significant differences were found between LA-RC $(M=94.434, \mathrm{SE}=.996)$ and LC-RA $(P>.05)$ or sham $(P>.05)$. For the vowel-consonant/parity task, LA-RC $(M=95.265$, $\mathrm{SE}=.675)$ significantly increased accuracy compared to sham $(M=87.451, \mathrm{SE}=2.858)(P=.009)$. No significant differences were found between LC-RA $(M=93.996, \mathrm{SE}=1.226)$ and both LA-RC and sham $(P>.05)$ (see Fig. 3). Nonetheless, LC-RA seems to reveal a trend of increasing accuracy when comparing to sham $(P=.060)$.

All other possible pairwise comparisons for the differential effects of the type of tDCS across tasks were not statistically significant $(P>.05)$ (see Table 1 for summary).

\section{Exploratory analysis of the novel targets}

The repeat/switch analysis only revealed main effects of repeat switch $\left(F(1,15)=146.498, P<.001, \eta_{\mathrm{p}}^{2}=.907, f=3.123\right.$, $\mathrm{pwr}=1.000)$, with the repeat trials being significantly faster than the switch ones $(P<.001)$, but no effects were found for the tDCS

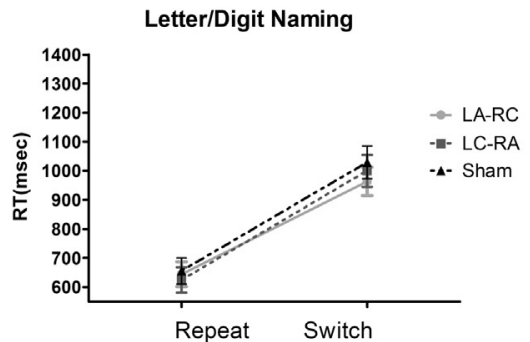

Letter/Digit Naming

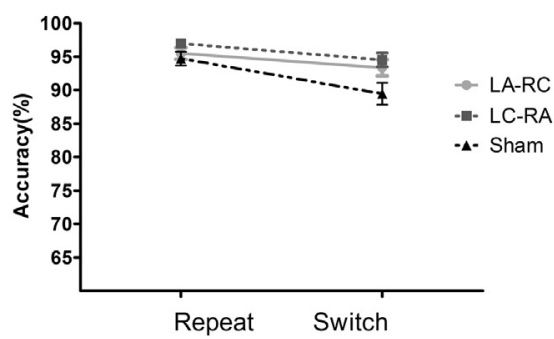

Vowel-Consonant/Parity

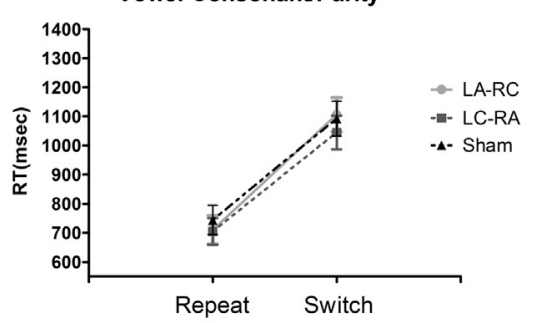

Vowel-Consonant/Parity

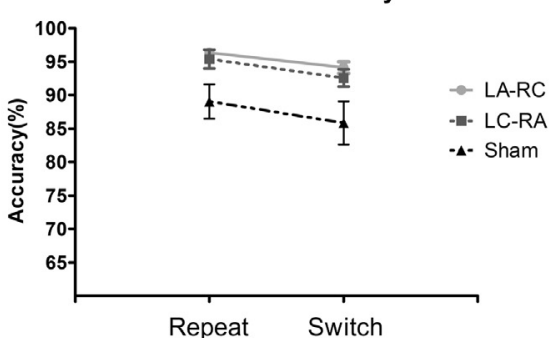

Novel: Vowel-Consonant/Parity

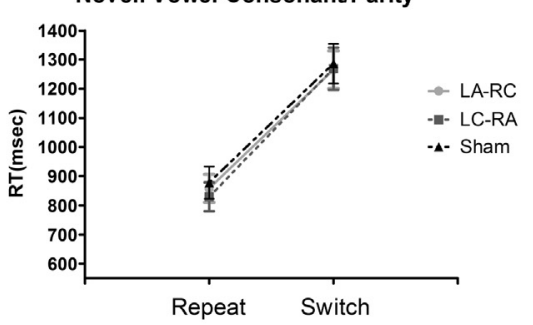

Novel: Vowel-Consonant/Parity

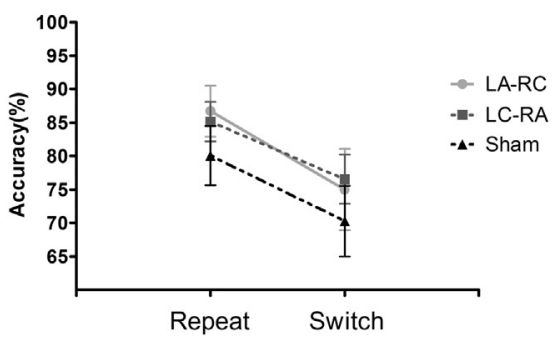

Figure 4. Switch-repeat analysis: RT and accuracy. The symbols represent the mean and the bars, the standard error (SE). RT: reaction time. Overall accuracy: percentage of correct responses. 
Summary of the results found in the post hoc pairwise comparisons

\begin{tabular}{|c|c|c|c|c|c|c|c|c|}
\hline & \multirow[b]{2}{*}{$\mathrm{tDCS}$} & \multicolumn{3}{|c|}{ Letter/digit naming task } & \multicolumn{3}{|c|}{ Vowel-consonant/parity task } & \multirow{2}{*}{$\frac{\text { Control }}{\text { RT }}$} \\
\hline & & LA-RC & LC-RA & Sham & LA-RC & LC-RA & Sham & \\
\hline Switch cost performance & LA-RC & & Increase & Increase & & Decrease & Decrease & \\
\hline Switch error & & & 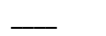 & - & & - & {[} & \\
\hline Overall accuracy & & & 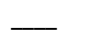 & $\overline{-}$ & & 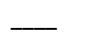 & Increase & $\ldots$ \\
\hline Switch cost performance & LC-RA & Decrease & & 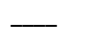 & Increase & & - & $\ldots$ \\
\hline Switch error & & 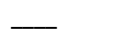 & & - & - & & {[} & \\
\hline Overall accuracy & & - & & Increase & 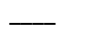 & & $\ldots$ & - \\
\hline
\end{tabular}

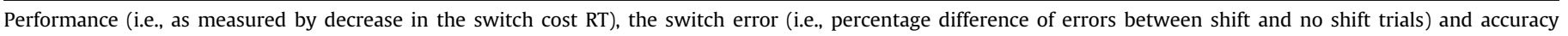
(i.e., percentages of correct responses in each task) according to the type of tDCS, for each task (letter/digit naming task, vowel-consonant/parity and control). Increase and decrease with statistically significant $P<.05$ - please note that all the comparisons are the effect of LA-RC or LC-RA tDCS of the left column.

$\left(F(2,30)=.119, P>.05, \eta_{\mathrm{p}}^{2}=.008, f=.090, \mathrm{pwr}=.067\right)$ nor interaction between tDCS and switch/repeat $(F(2,30)=.361, P>.05$, $\left.\eta_{\mathrm{p}}^{2}=.024, f=.157, \mathrm{pwr}=.103\right)$.

There were no significant effects of tDCS on the novel targets in terms of switch cost RT $\left(F(2,30)=.361, P>.05, \eta_{\mathrm{p}}^{2}=.024, f=.157\right.$, $\mathrm{pwr}=.103)$, accuracy $\left(F(2,30)=.452, P>.05, \eta_{\mathrm{p}}^{2}=.029, f=.173\right.$, pwr $=.117)$ or switch cost errors $\left(F(2,30)=.291, P>.05, \eta_{\mathrm{p}}^{2}=.019\right.$, $f=.139$, pwr $=.092$ ).

\section{Discussion}

This study demonstrates that cross-hemispheric driven tDCS over the PFC can change switch cost performance, as well as overall accuracy on switching tasks. Moreover, depending on the task, the tDCS modulation produces asymmetric effects in inter-hemispheric interactions. On the letter/digit naming task, LA-RC tDCS decreased RT switch costs (thus increasing performance), whereas LC-RA tDCS increased the overall accuracy as measured by the percent of correct responses. At the same time, on the vowel-consonant/ parity task, LA-RC tDCS decreased performance (by reducing the switch cost RT) compared to LC-RA tDCS, but increased the accuracy compared to sham. Moreover, LA-RC tDCS is the only one that seems to have significant and distinct effects depending on the task: increasing performance in the letter/digit naming task and decreasing in the vowel-consonant/parity task, suggesting asymmetric involvement of the $\mathrm{RH}-\mathrm{LH}$ interaction.

Participants in the three tDCS conditions did not show statistically significant differences in simple reaction time, choice reaction time or overall accuracy. There were no significant effects of tDCS on the amount of time required to respond to the stimulus or the choice reaction time, where the participant must discriminate each stimulus and perform a response selection. These data suggest that the differences found in this study may not be due to motor or response speed changes. Nonetheless, the simple and choice RT tasks were not repeated at the end of the experiment and this could be seen as a potential limitation of this study.

Even though both tasks rely on the same S-R mappings, the different instructions seem to have impacted the results for response speed (performance) but not overall accuracy. This effect was present without the novel targets (that were analyzed separately). This systematic increase in RT has been already showed in studies as a function of memory load [21]. Also, as expected, repeat trials were faster than switch trials (e.g., [9]). At least partially, the conditions presented in this study can be related to different working memory loads, especially due to the different levels of cognitive control mechanisms required to successfully complete the tasks. It is important to note, that the novel targets had the highest RTs in this experiment, but were not affected by tDCS. It is not possible to speculate why tDCS had no effect in these targets, mainly due to a lack of statistical power in the present design.
Cross-hemispheric driven tDCS over the PFC was found to have specific effects depending on the switching task. The results found in the letter/digit naming task, that LA-RC improves switch cost performance, have already been shown in another study [22], where participants in a probabilistic guessing task were faster at choosing the most frequent alternative. At the same time, in this study, LC-RA tDCS increased accuracy. Moreover, LC-RA showed also a trend of increasing accuracy in the vowel-consonant/parity task. Although the particular mechanism underlying this effect is not clear, this same result has been associated to a broader effect of this LC-RA tDCS in decision making, found in studies related to risk taking [23] or food craving [24]. Thus, the data in this study supports the claim that this particular electrode montage might be related to the modulation of circuits related to decision making [23-25].

The present data is consistent with the different strategies of information processing over the left and the right hemispheres (e.g., $[11,13])$. One possibility is that in the letter/digit naming task, the possible responses could be easily predetermined (the cue will indicate which target will be selected, by pressing the associated key). The activity change toward the LH (with the LA-RC tDCS) improved switch cost performance, and this is consistent with the propensity of the LH to better deal with determinate, precise and unambiguous representations [22]. Conversely, LC-RA tDCS changed the activity toward the $\mathrm{RH}$, and despite not producing significant RT differences in switch costs, it improved the overall accuracy within the task, possibly by modulating circuits involved in decision making [23-25].

The vowel-consonant/parity task requires recognition and, afterward, a simple judgment prior to response selection (i.e., the cue will indicate to which target the participant will make one of two possible judgments, which will then determine which key will be selected). In this more demanding (or even "ambiguous" condition due to inclusion of a secondary task prior to response selection) situation, LA-RC tDCS decreased performance. The LH tendency to "overinterpret" information (e.g., [11,26,27]) may be responsible for slower performance in this more demanding situation, which has been found in previous studies but was not related to risk-taking behaviors [23]. This "overinterpretation" could lead to slower performance but increased accuracy. Studies suggest that a lesion to the RH PFC will impair its inhibitory function and then the LH PFC-imposed interpretation will be rendered determinate (e.g., $[11,13,26])$. In our study, the LH tendency to fill in the gaps does not seem to lead to premature conclusions as demonstrated by the increased accuracy. One possibility here is that there were no tDCS effects in the inhibitory function of the RH. Modeling studies with tDCS already demonstrated that the peak of the current is induced under the electrode $[28,29]$, and a possibility is that the right inferior frontal gyrus (rIFG), responsible for this functional inhibition [30], was not affected by the tDCS. This possibility gains further 
support as tDCS over the rIFG is capable of neuromodulating this inhibitory functioning [31].

Another important factor is if attention plays a significant role in our results. Although the DLPFC is involved in attention-related task processing, other areas such as dorsal parietal areas seem to be more critical for modulating attentional resources. In fact, research has shown that intentional shifts in visual attention are dependent on a bi-hemispheric network consisting of DLPFC and parietal regions [32,33]. This network is thought to be involved in top-down selection of stimuli, thus guiding goal-directed behavior [33]. In this context, the level of difficulty of a task that demands additional prefrontal engagement (for instance, the vowel-consonant/parity task) may require more resources (as revealed by the increase in the RTs), thus decreasing parietal activation and attention span; therefore, the cathodal tDCS over the DLPFC may have reversed this change in activation, improving accuracy by a secondary effect on attentional networks. Nonetheless, studies targeting several levels of attentional demands, and combining tDCS and fMRI are needed in order to test this hypothesis.

This study suggests that the inter-hemispheric balance between the homotopic PFC may be important in task switching. The data from this study are consistent with the assumption that more than a nonspecific switching area, there could be specific interhemispheric activity balance in the PFC (and other regions) involved in task switching that future studies should try to determine. One of those, for instance, will be the coordinating role of the inferior parietal cortex that in task switching seems to precede the role of the PFC [34].

The present study has several limitations. First, we cannot conclude if the effects are due to the anodal or cathodal tDCS or if they are due to anodal/cathodal interaction effects. The results found in this exploratory study need to be replicated with larger samples. Also, we cannot dismiss the possibility of carryover effects between sessions (despite the washout period), so future research should use weekly periods between sessions or, preferably, between-subjects designs. Although the tasks employed in this study worked as designed, they need to be further replicated. Also, future studies with functional magnetic resonance (fMRI) combined with online tDCS should be performed to test the asymmetric involvement of right and left hemispheres in those tasks, as well as the cross-hemispheric tDCS effects in other brain regions. Future studies should also test the possible effects of crosshemispheric driven tDCS on the parietal cortex, as studies with tDCS [35] and with single cell recording suggest the specialization of the parietal region during both the preparation and behavioral execution of switching tasks (i.e., parietal cortex as a suitable area for potential neuromodulation) [36]. Future studies should also focus on the inferior frontal gyrus, due to its crucial role in inhibition [30] and in novel target detection [37]. Also, switching tasks seems to include both an endogenous preparation component (i.e., without the presence of an external stimulus) as well as an exogenous executive component (i.e., a response adjustment to that stimulus) (e.g., [38,39]). Thus, future studies should use tasks specifically designed for each component.

In summary, the present data shows that increasing the activity of the left hemisphere, while decreasing the activity in the right one increases switch cost performance but only for the letter/digit naming task. For the vowel-consonant/parity task, LA-RC tDCS decreased switch cost performance, suggesting that activity increase in the $\mathrm{LH}$, while decreasing the activity in the homotopic contralateral region impairs performance; however, this strategy enhances accuracy. More studies manipulating the frequency of targets involved in the response selection generation, with larger samples sizes, as well as testing the cross-hemispheric effects of tDCS over the PFC and the parietal cortex in other cognitive functions are needed in order to fully understand the present results.

\section{Acknowledgments}

The author's thank Fernando Macedo for the technical support to the present work.

\section{References}

[1] Brass M, von Cramon DY. Decomposing components of task preparation with functional magnetic resonance imaging. J Cogn Neurosci 2004;16(4):609-20.

[2] Monsell S. Task switching. Trends Cogn Sci 2003;7(3):134-40.

[3] Dreher JC, Grafman J. Dissociating the roles of the rostral anterior cingulate and the lateral prefrontal cortices in performing two tasks simultaneously or successively. Cereb Cortex 2003;13(4):329-39.

[4] Nagahama Y, Okada T, Katsumi Y, Hayashi T, Yamauchi H, Oyanagi C, et al. Dissociable mechanisms of attentional control within the human prefrontal cortex. Cereb Cortex 2001;11(1):85-92.

[5] Ravizza SM, Carter CS. Shifting set about task switching: behavioral and neural evidence for distinct forms of cognitive flexibility. Neuropsychologia 2008; 46(12):2924-35.

[6] Barber AD, Carter CS. Cognitive control involved in overcoming prepotent response tendencies and switching between tasks. Cereb Cortex 2005;15(7): 899-912.

[7] Aron AR, Monsell S, Sahakian BJ, Robbins TW. A componential analysis of taskswitching deficits associated with lesions of left and right frontal cortex. Brain 2004;127(Pt 7):1561-73.

[8] Sohn MH, Ursu S, Anderson JR, Stenger VA, Carter CS. The role of prefrontal cortex and posterior parietal cortex in task switching. Proc Natl Acad Sci U S A 2000;97(24):13448-53.

[9] Leite J, Carvalho S, Fregni F, Gonçalves ÓF. Task-specific effects of tDCSinduced cortical excitability changes on cognitive and motor sequence set shifting performance. PLoS One 2011;6(9):e24140.

[10] D’Esposito M, Cooney JW, Gazzaley A, Gibbs SE, Postle BR. Is the prefrontal cortex necessary for delay task performance? Evidence from lesion and FMRI data. J Int Neuropsychol Soc 2006;12(2):248-60.

[11] Gazzaniga MS. Cerebral specialization and interhemispheric communication: does the corpus callosum enable the human condition? Brain 2000;123(Pt 7): 1293-326.

[12] Goel V, Vartanian O. Dissociating the roles of right ventral lateral and dorsal lateral prefrontal cortex in generation and maintenance of hypotheses in setshift problems. Cereb Cortex 2005;15(8):1170-7.

[13] Goel V, Tierney M, Sheesley L, Bartolo A, Vartanian O, Grafman J. Hemispheric specialization in human prefrontal cortex for resolving certain and uncertain inferences. Cereb Cortex 2007;17(10):2245-50.

[14] Fregni F, Boggio P, Nitsche M, Bermpohl F, Antal A, Feredoes E, et al. Anodal transcranial direct current stimulation of prefrontal cortex enhances working memory. Exp Brain Res 2005;166(1):23-30.

[15] Rypma B, D’Esposito M. The roles of prefrontal brain regions in components of working memory: effects of memory load and individual differences. Proc Natl Acad Sci U S A 1999;96(11):6558-63.

[16] Nitsche MA, Paulus W. Sustained excitability elevations induced by transcranial DC motor cortex stimulation in humans. Neurology 2001;57(10): 1899-901.

[17] Ardolino G, Bossi B, Barbieri S, Priori A. Non-synaptic mechanisms underlie the after-effects of cathodal transcutaneous direct current stimulation of the human brain. J Physiol 2005;568(2):653-63.

[18] Boggio PS, Fregni F, Valasek C, Ellwood S, Chi R, Gallate J, et al. Temporal lobe cortical electrical stimulation during the encoding and retrieval phase reduces false memories. PLoS One 2009;4(3):e4959.

[19] Nitsche MA, Paulus W. Excitability changes induced in the human motor cortex by weak transcranial direct current stimulation. J Physiol 2000;527(3): 633-9.

[20] Jasper $H$. The ten-twenty electrode system of the International Federation. Electroencephalogr Clin Neurophysiol Suppl 1958;10(1):371-5.

[21] Jensen O, Gelfand J, Kounios J, Lisman JE. Oscillations in the alpha band (9-12 $\mathrm{Hz}$ ) increase with memory load during retention in a short-term memory task. Cereb Cortex 2002;12(8):877-82.

[22] Hecht D, Walsh V, Lavidor M. Transcranial direct current stimulation facilitates decision making in a probabilistic guessing task. J Neurosci 2010;30(12): 4241-5.

[23] Fecteau S, Knoch D, Fregni F, Sultani N, Boggio P, Pascual-Leone A. Diminishing risk-taking behavior by modulating activity in the prefrontal cortex: a direct current stimulation study. J Neurosci 2007;27(46):12500-5.

[24] Fregni F, Orsati F, Pedrosa W, Fecteau S, Tome FA, Nitsche MA, et al. Transcranial direct current stimulation of the prefrontal cortex modulates the desire for specific foods. Appetite 2008;51(1):34-41.

[25] Boggio PS, Sultani N, Fecteau S, Merabet L, Mecca T, Pascual-Leone A, et al. Prefrontal cortex modulation using transcranial DC stimulation reduces alcohol craving: a double-blind, sham-controlled study. Drug Alcohol Depend 2008;92(1-3):55-60. 
[26] Wolford G, Miller MB, Gazzaniga M. The left hemisphere's role in hypothesis formation. J Neurosci 2000;20(6):RC64.

[27] Walsh V. Hemispheric asymmetries: a brain in two minds. Curr Biol 2000; $10(12): \mathrm{R} 460-2$.

[28] Wagner T, Fregni F, Fecteau S, Grodzinsky A, Zahn M, Pascual-Leone A. Transcranial direct current stimulation: a computer-based human model study. NeuroImage 2007;35(3):1113-24.

[29] Miranda PC, Lomarev M, Hallett M. Modeling the current distribution during transcranial direct current stimulation. Clin Neurophysiol 2006;117(7): 1623-9.

[30] Aron AR, Robbins TW, Poldrack RA. Inhibition and the right inferior frontal cortex. Trends Cogn Sci 2004;8(4):170-7.

[31] Jacobson L, Javitt DC, Lavidor M. Activation of inhibition: diminishing impulsive behavior by direct current stimulation over the inferior frontal gyrus. J Cogn Neurosci 2011;23(11):3380-7.

[32] Husain M, Nachev P. Space and the parietal cortex. Trends Cogn Sci 2007; 11(1):30-6.
[33] Corbetta M, Patel G, Shulman GL. The reorienting system of the human brain: from environment to theory of mind. Neuron 2008;58(3):306-24.

[34] Bode S, Haynes JD. Decoding sequential stages of task preparation in the human brain. Neurolmage 2009;45(2):606-13.

[35] Stone DB, Tesche CD. Transcranial direct current stimulation modulates shifts in global/local attention. NeuroReport 2009;20(12):1115-9.

[36] Kamigaki T, Fukushima T, Miyashita Y. Neuronal signal dynamics during preparation and execution for behavioral shifting in macaque posterior parietal cortex. J Cogn Neurosci 2011;23(9):2503-20.

[37] Yamasaki H, LaBar KS, McCarthy G. Dissociable prefrontal brain systems for attention and emotion. Proc Natl Acad Sci U S A 2002;99(17):11447-51.

[38] Ruthruff E, Remington RW, Johnston JC. Switching between simple cognitive tasks: the interaction of top-down and bottom-up factors. J Exp Psychol Hum Percept Perform 2001;27(6):1404-19.

[39] Rubinstein JS, Meyer DE, Evans JE. Executive control of cognitive processes in task switching. J Exp Psychol Hum Percept Perform 2001;27(4): 763-97. 